\title{
DESIGN AND MANUFACTURE OF A PROTOTYPE UNDULATOR FOR THE LCLS PROJECT*
}

\author{
E.R Moog†, R.J. Dejus, P.K. Den Hartog, E. Gluskin, E. Trakhtenberg, I. Vasserman, \\ Advanced Photon Source, Argonne National Lab, Argonne, IL 60439, USA \\ V. Tcheskidov, and N. Vinokurov, Budker Institute of Nuclear Physics, Novosibirsk, Russia
}

\begin{abstract}
The goal of the Linac Coherent Light Source (LCLS) project is to build a free-electron laser (FEL) that will produce $\mathrm{x}$-rays of $1.5 \AA$ wavelength by sending a 14.35 $\mathrm{GeV}$ electron beam from the Stanford Linear Accelerator through a line of undulators. The undulator line will consist of a series of 3.4-m-long undulator sections, with diagnostics inserted between the sections. The design for an undulator section has been completed, and a prototype is under construction at the Advanced Photon Source. The undulator will be a fixed-gap device, with a magnetic pole gap of $6 \mathrm{~mm}$ and a period of $30 \mathrm{~mm}$. The strongback is machined with high accuracy from a solid, forged Ti bar. Titanium was chosen for its low thermal expansion and for its strength and low density, which will reduce the weight and the deflection of the strongback under gravity. Stringent magnetic requirements demand a stable undulator, and the fixed gap will help achieve it.
\end{abstract}

\section{INTRODUCTION}

The undulator line for the LCLS will consist of 33 separate undulator segments. Focusing quadrupoles, in a FODO lattice, and electron beam diagnostics will be located in the breaks between undulator segments. After every third undulator segment, the break will be longer so that photon diagnostics can be installed as well. Thus, taking the alternating focusing and defocusing quadrupoles into account, the 'super-period' length before the undulator line repeats itself is six undulators.

The undulator segments are planar permanent-magnet hybrid arrays, using vanadium permendur poles and Nd-Fe$B$ permanent magnets, with a period of $30 \mathrm{~mm}$ and a nominal magnetic gap of $6 \mathrm{~mm}$. The actual magnetic gap will be adjusted to achieve an effective $\mathrm{K}$ of 3.71 , or an effective $\mathrm{B}$ of $13250 \mathrm{G}$. The maximum outside dimension of the vacuum chamber is $5.6 \mathrm{~mm}$ in order to allow for the 6-mm undulator magnetic gap. The grade of magnet material selected is one with very high coercivity to increase the magnets' resistance to radiation-induced demagnetization. The poles and magnets will be rectangular rather than wedged, to help keep the mechanical design and fabrication of the magnetic structure more straightforward.

\section{UNDULATOR REQUIREMENTS}

The x-ray output of the LCLS will be noisier than what synchrotron users have come to expect, because noisiness

\footnotetext{
*Supported by the U.S. D.O.E., under Contract \# W-31-109-ENG-38 and DE-AC03-76SF00515.

$\dagger$ moog@aps.anl.gov
}

is intrinsic to the self-amplified spontaneous emission (SASE) process [1]. The noise level can be reduced somewhat by operating the FEL at saturation. The total length available for the undulator line is limited, so a goal in setting the design requirements of the undulator line is to minimize the FEL gain length, which will also minimize the total length of undulator needed to reach saturation. A tolerance budget has been worked out for the parameters that affect the saturation length, assuming simultaneous worst cases for all parameters. The overall tolerances for the undulator line were then used to determine tolerances for a single undulator section. These tolerances are: the trajectory walk-out from a straight line must not exceed 2 microns in either transverse direction; the reduction in spectral intensity of the zero-angle radiation as compared to an ideal undulator must not exceed 4\%; the calculated electron phase deviation from the design value must be less than $10^{\circ}$; and the undulator median plane must be defined (and after that aligned) with an accuracy better than 50 microns vertically. These tolerances, combined, give an increase in the gain length by $3 \%$. It is encouraging that these tolerances are realistic; they were met by the undulators that were tuned magnetically for the Advanced Photon Source (APS) FEL, and the tuning techniques developed for the $3.3-\mathrm{cm}$-period APS FEL undulators should transfer directly to the 3.0cm-period LCLS undulators.

\section{MAGNETIC DESIGN}

Implicit in the above tolerances is the need for the magnetic field strength to be uniform along the length of the undulator line. Although some adjustment of the phasing is possible at the ends of the undulators, that cannot correct variations within an undulator. Simulations using the code RON [2] to change the strength of one undulator segment found that the effect of nonuniform field strengths becomes significant when undulator-to-undulator variations reach $\Delta \mathrm{B} / \mathrm{B}=1.3 \times 10^{-4}$. This error in magnetic field would be obtained if there were an error in the gap of the undulator by $1.2 \mu \mathrm{m}$. This sensitivity to magnetic field strength imposes demanding requirements on the mechanical stability of the undulator magnetic gap.

This sensitivity to field strength also requires that attention be paid to thermal effects. The remanent field of the permanent magnets decreases with increasing temperature by $0.1 \% /{ }^{\circ} \mathrm{C}$. Thus, in the absence of thermal expansion, the requirement for temperature uniformity along the undulator line would be of the order of $0.1^{\circ} \mathrm{C}$, 
i.e., unrealistic. Instead, the undulator is designed so that thermal expansion causes the gap to close slightly to compensate for the decrease in remanence.

Another concern with the LCLS undulators is the likelihood of loss of magnetic field strength due to radiation-induced demagnetization of the permanent magnet blocks. The high field strength of $\mathrm{NdFeB}$ magnets is needed, but $\mathrm{NdFeB}$ is sensitive to radiation. Demagnetization has been seen in the undulators at the European Synchrotron Radiation Facility [3], so it is a well-recognized risk. Resistance to radiation damage can be enhanced by the choice of magnet material and by the magnetic design of the undulator. The likelihood of radiation-induced demagnetization has been found to increase when the demagnetizing field experienced by the magnet block is stronger [4]. Therefore, a larger margin between the demagnetizing field experienced by the magnet block and the demagnetizing field at which the block will demagnetize is expected to give a higher resistance to radiation-induced demagnetization. To get the larger margin, we 1) chose a grade of magnet material with a high coercivity, and 2) designed the magnetic structure to keep the demagnetizing field down. The grade of magnet material chosen is N39SH from either ShinEtsu or Sumitomo Special Metals. This grade has a high coercivity of $21 \mathrm{kOe}$ and does not sacrifice magnetic remanence as compared to the older $\mathrm{N} 38 \mathrm{H}$ grade that has been used in many insertion devices, including most of those at the APS. During the magnetic design process, the localized demagnetizing field experienced by different parts of the magnet blocks was examined over the full range of intended gaps. A goal in the design was to maximize the on-axis field of the undulator while ensuring that the demagnetizing field seen by the magnets is not excessive at any gap that the device will experience.

Straightness of the trajectory is a significant requirement for the undulators, and a proper design for the ends of the undulators will help keep the trajectory straight. The strengths of the poles at the ends of the undulators will go in the sequence $0.25,0.75,1$. This gives an entrance into (and exit from) the undulator that has no angle kick and no trajectory offset.

\section{MECHANICAL DESIGN}

A photo of a short model for an undulator segment is shown in Fig. 1. For the full-length undulator, the Cshaped undulator support is made from a solid 3.4-m-long titanium bar that has a diameter of approximately 305 $\mathrm{mm}$. Titanium was chosen as the material due to its low specific weight and low thermal expansion coefficient. The low specific weight will lighten the structure and thus decrease the sag of the undulator between supports; deflections of only a few microns are anticipated. Titanium's low thermal expansion coefficient will minimize thermally induced deflections caused by variations in the tunnel temperature. The top and bottom magnetic jaws fit into the precise window machined along

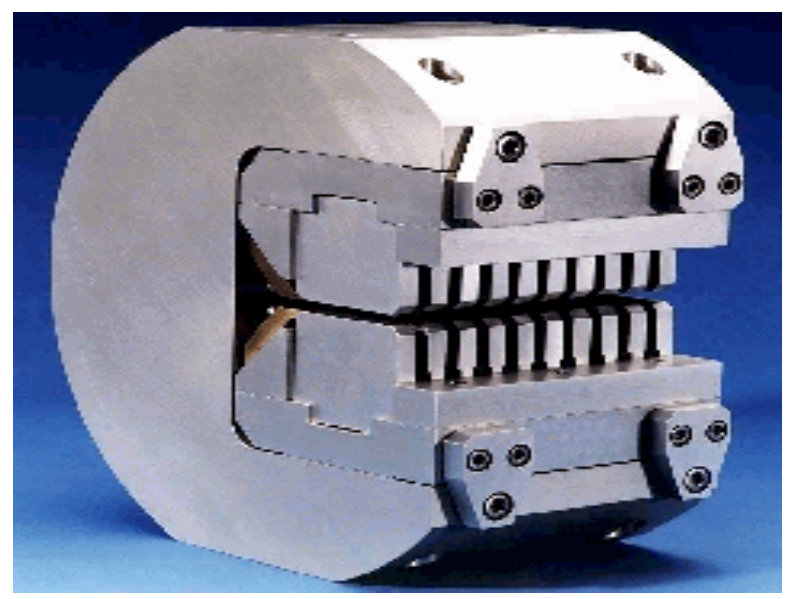

Figure 1: Short model (9 poles, 10 magnets) of the assembled LCLS undulator design. The C-shaped support is of titanium.

the entire length of the bar. The magnets and poles are assembled on an Al base plate, which sits against the $\mathrm{Ti}$ support. The thermal expansion of the $\mathrm{Al}$ base plate is somewhat larger than for $\mathrm{Ti}$, so the magnetic gap of the undulator will decrease slightly with increasing temperature to compensate for the loss in remanence.

Figures 2 and 3 show some of the details of how the magnetic structure is held, and Fig. 4 shows one jaw removed from the short model. The magnetic structure is designed in such a way that only one clamp is required to hold each pole and each magnet, thus leaving one side of the structure open for the insertion of side shims and for easier magnetic measurements.

The poles of the prototype undulators are located in precisely machined grooves located in the two sidebars. In order to achieve the maximum peak field, the magnets extend beyond the poles in three directions - on both sides, and in the direction away from the magnetic gap. There is no space on the gap side of the pole to clamp the pole into place, so the poles have nonmagnetic "ears" added to their sides for clamping. The ears are made of titanium alloy because its thermal expansion is nearly the

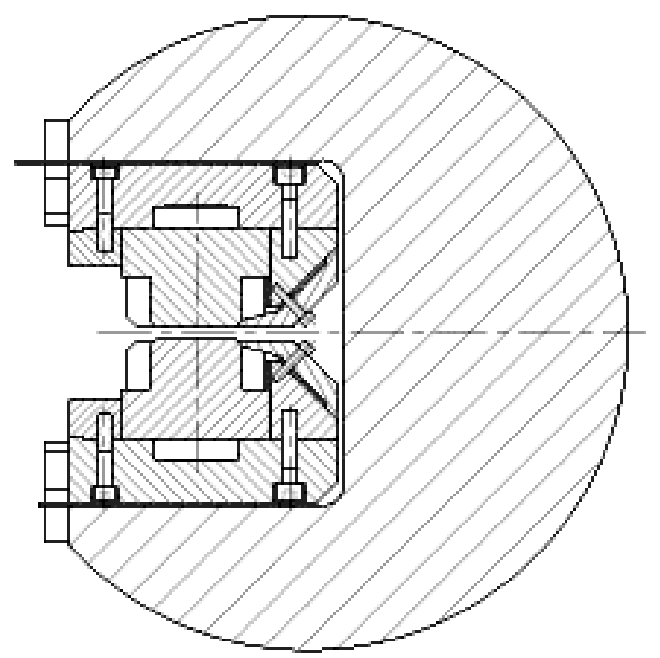

Figure 2: View of the undulator, through a pole. 


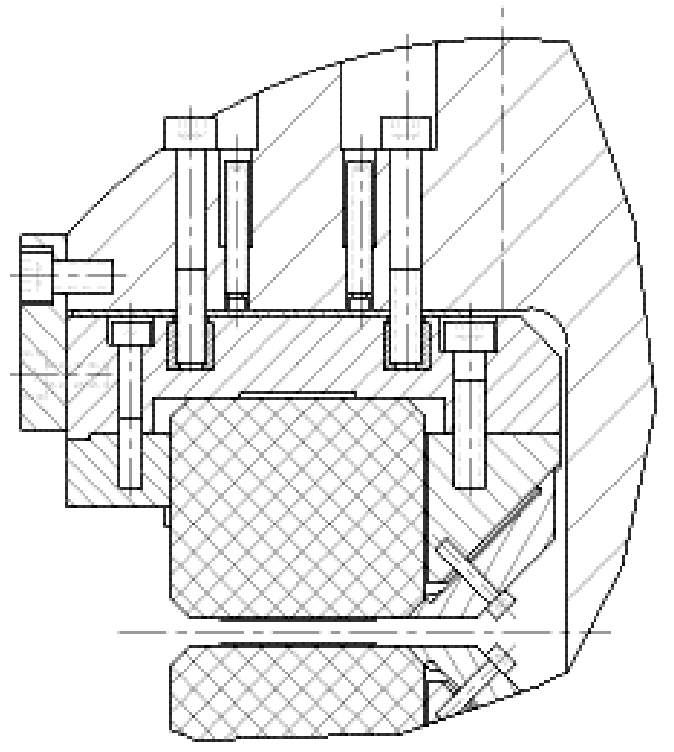

Figure 3: Enlarged undulator view, through a magnet. same as that of the vanadium permendur pole material. They are fastened to the pole, then undergo final grinding and heat-treating along with the poles (see Fig. 5).

The magnetic structure of each jaw assembly consists of five blocks placed end to end with three long blocks approximately $1 \mathrm{~m}$ long in the middle and a shorter block at each end. Precision shims of various thicknesses can be placed between the $\mathrm{Ti}$ bar and the jaw for fine adjustment of the undulator gap. Shims in 2 micron increments are being made for this by nickel-plating brass shims. The same result can also be obtained using "pushpull" screws.

The magnetic structure can be additionally tuned using low-carbon steel screws, referred to as side shims, which can be screwed in towards the poles, as shown in Fig. 6 . These side shims can be installed anywhere along the length of the undulator as required for precise tuning.

The two end blocks for each jaw can also be bent slightly, up to 80 microns per jaw with submicron resolution, in order to adjust the phasing at the end of the undulator. For this purpose, four PZT translators are housed in holes within the titanium core.
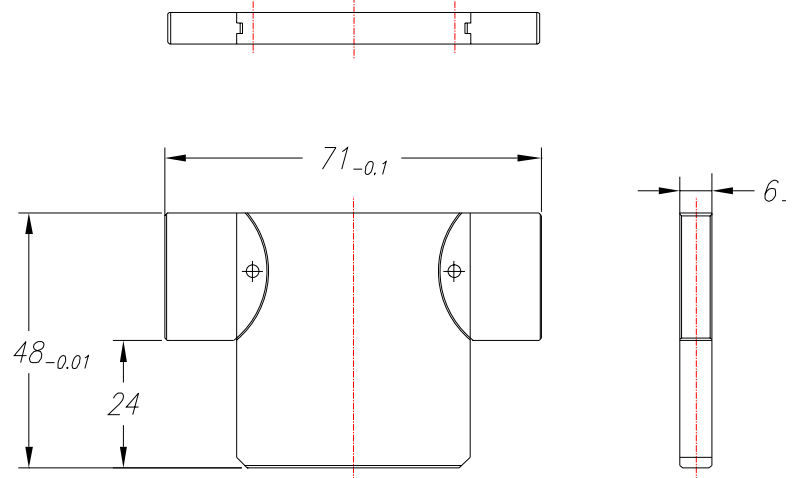

Figure 5: Ti ears are attached to the vanadium permendur poles so the poles can be clamped in place.

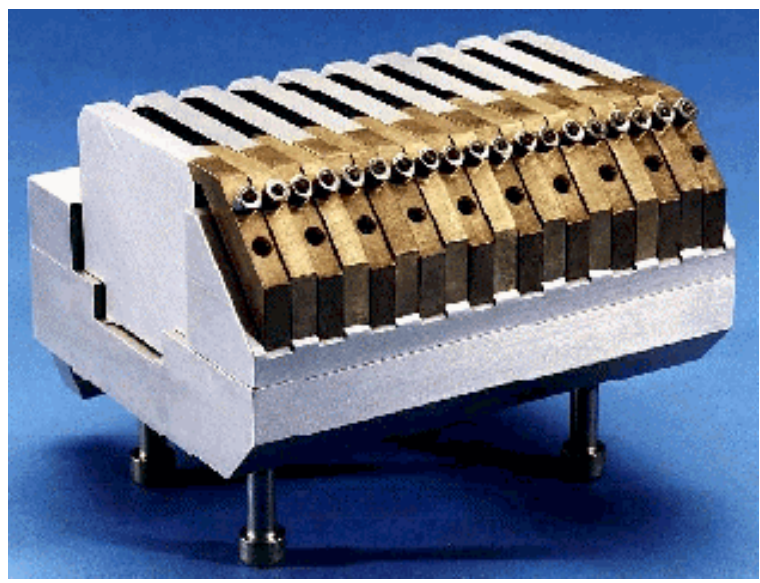

Figure 4: A jaw of the magnetic structure removed from the short model.

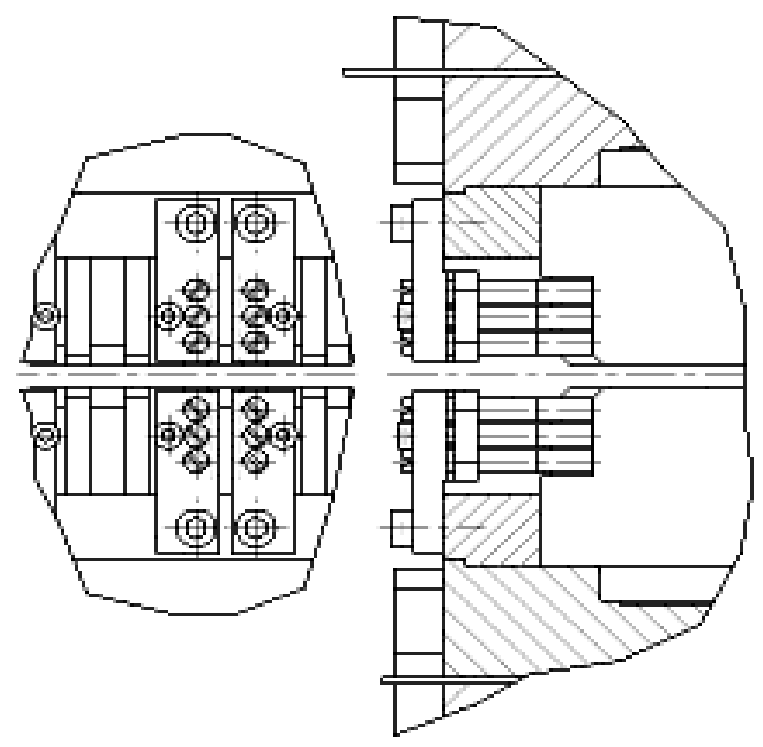

Figure 6: Side shims for magnetic tuning.

\section{SUMMARY}

A full-sized, $3.4 \mathrm{~m}$ long prototype undulator segment for the LCLS is under construction at the APS. The undulator design emphasizes stability of the magnetic field, but still allows the magnetic field strength to be adjusted so it can be made uniform for all undulator segments. In addition to provisions for the usual undulator magnetic tuning, the gap of the end blocks is adjustable so the phasing between undulators will be adjustable.

\section{REFERENCES}

[1] K.-J. Kim, Nucl. Instrum. Meth. A 393, 167 (1997); R. Bonifacio et al., Phys. Rev. Lett. 73, 70 (1994).

[2] R.J. Dejus, O.A. Shevchenko, and N.A. Vinokurov, Nucl. Instrum. Meth. Phys. Res. A 445, 19 (2000).

[3] J. Chavanne, P. Elleaume, and P. Van Vaerenbergh, ESRF Machine Tech. Note 1-1996/ID (Jan. 1996).

[4] R.D. Brown and J.R. Cost, J. Appl. Phys. 63, 3537 (1988) and IEEE Trans. Magn. 25, 3117 (1989). 